\author{
ARTICULO \\ Revista Derecho - Año 3 edicion 5131 - 144
}

Web: http://revistas.unap.edu.pe/rd E-mail.com: revistaderecho@unap.edu.pe ISSN 2313-6944

\title{
ESTUDIO SOBRE EL DELITO DE INCUMPLIMIENTO DE LA OBLIGACION ALIMENTARIA DESDE LAS PERSPECTIVAS DE LAS ESCUELAS JURIDICO-PENALES
}

\author{
Huallpa Barreda, Aleshka J. \\ Laqui Huanacune, Dania \\ Pumabualcca Coila, Danitza \\ Ticona Valder, Karen S. \\ Quispe Ruelas, Hosen. E.
}

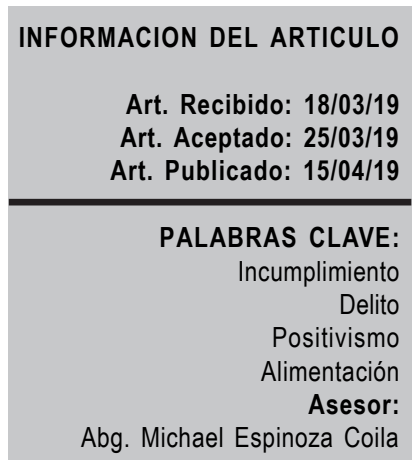

Abg. Michael Espinoza Coila

\section{RESUMEN}

La escuela jurídico penal, de acuerdo con Jiménez A, es el cuerpo orgánico de concepciones contrapuestas sobre la legitimidad del derecho de pensar sobre la naturaleza del delito y sobre el fin de las sanciones; para Ferri la escuela positiva consiste en lo siguiente estudiar al delito, primero en su génesis natural, y después en sus efectos jurídicos, para adaptar jurídicamente diversos remedios a las varias causas que lo producen los que, en consecuencia serán eficaces, presentando la escuela clásica trece postulados; las críticas contra las escuelas clásica y positivista han dado nacimiento a escuelas eclécticas que mantienen principios de la clásica y toman otros de la positivista, situándose en un término medio 


\section{STUDY ON THE CRIME OF NON-COMPLIANCE OF FOOD OBLIGATION FROM THE PERSPECTIVES OF THE JURIDICAL-PENAL SCHOOLS}

\begin{tabular}{|c|c|}
\hline ARTICLE INFO & ABSTRACT \\
\hline $\begin{array}{r}\text { Article Received: } 18 / 03 / 19 \\
\text { Article Accepted: } 25 / 03 / 19 \\
\text { Article Published: } 15 / 04 / 19\end{array}$ & $\begin{array}{l}\text { The penal legal school, according to Jiménez A, is the organic body } \\
\text { of opposing conceptions about the legitimacy of the right to think } \\
\text { about the nature of crime and about the end of sanctions; for Ferri }\end{array}$ \\
\hline $\begin{array}{r}\text { KEY WORDS: } \\
\text { Breach } \\
\text { Crime } \\
\text { Positivism } \\
\text { Food } \\
\text { Adviser: } \\
\text { Abg. Michael Espinoza Coila }\end{array}$ & $\begin{array}{l}\text { its natural genesis, and then in its juridical effects, in order to legally } \\
\text { adapt various remedies to the various causes that produce it, which } \\
\text { will consequently be effective, presenting the classical school thirteen } \\
\text { postulates; Criticisms against the classical and positivist schools have } \\
\text { given birth to eclectic schools that maintain classical principles and } \\
\text { take others from the positivist one, standing at a medium level }\end{array}$ \\
\hline
\end{tabular}

132 Revista Derecho-5 (2019) 


\section{INTRODUCCIÓN}

El rápido desarrollo de las escuelas jurídico penales en el siglo XX, se debió a los debates y confrontaciones, que ocurrieron entre las dispersas escuelas jurídico penales. Algunos de los avances más importantes que se obtuvieron de la pugna de esas escuelas, consistió en la delimitación de los campos, en la precisión de métodos y en la colaboración entre profesionales, puesto que anteriormente trabajaban desperdigadas

\section{OBJETIVOS}

- Desarrollar los parámetros y/o concepciones de las escuelas jurídico penales, en cuanto al delito de incumplimiento de obligación alimentaria.

- Determinar el grado de influencia de las escuelas jurídico penales en el delito de incumplimiento de obligación alimentaria.

Diagnosticar si el delito de incumplimiento de obligación alimentaria, es pertinentemente considerado como tal.

\section{LAS ESCUELAS JURIDICO PENALES:}

De acuerdo con Jiménez A. (1950), las escuelas jurídicas penales son: «el cuerpo orgánico de concepciones contrapuestas sobre la legitimidad del derecho de pensar sobre la naturaleza del delito y sobre el fin de las sanciones».

Sainz Cantero (1990) define a las escuelas jurídicas penales en un sentido más amplio: «la dirección de pensamiento que tiene una determinada dirección, trabaja con un método peculiar y responde a unos determinados presupuestos filosóficos-penales». (Sainz, 1990)

\subsection{ESCUELA POSITIVA:}

La aparición del positivismo fue consecuencia del auge alcanzado por las ciencias naturales en los estudios filosóficos del siglo XIX, y se hizo sentir en todas las disciplinas incluyendo al derecho, la psiquiatría, la criminología y la psicología. Es una postura filosófica que tuvo un impacto y por tanto una influencia enorme en el campo de lo científico y por supuesto la búsqueda del conocimiento comprobable y válido. El positivismo está estrechamente ligado a la búsqueda metódica sustentada en lo experimental, rechazando nociones religiosas, morales, apriorísticas o conceptos abstractos, universales o absolutos. Lo que no fuese demostrable materialmente, por vía de experimentación reproducible, no podía ser científico.

Revista Derecho - 5 (2019) 133 
APORTES:

FERRI, (1887): La escuela positiva consiste en lo siguiente: estudiar al delito, primero en su génesis natural, y después en sus efectos jurídicos, para adaptar jurídicamente diversos remedios a las varias causas que lo producen los que, en consecuencia serán eficaces. (Ferri, Los nuevos horizontes del Derecho y del Procedimiento Penal, 1887)

FERRI, (1933): La escuela criminal positiva no consiste únicamente, en el estudio antropológico del criminal, pues constituye una renovación completa, un cambio radical de método científico en el estudio de la patología socio criminal, y de los que hay de más eficaz entre los remedios sociales y jurídico que nos ofrece. La escuela ha hecho de ello una ciencia de observación positiva, que, fundándose en la antropología, la psicología y la estadística criminal, el derecho penal y los estudios penitenciarios, llega a ser la ciencia sintética, que él mismo, la llamo sociología criminal, y así esta ciencia, aplicando el método positivo al estudio del delito, del delincuente y del medio, no hace otra cosa que llevar a la ciencia criminal clásica el soplo vivificador de las últimas e irrefragables conquistas hechas por la ciencia del hombre y de la sociedad, renovada por las doctrinas evolucionistas. (Ferri, 1933).

134 Revista Derecho-5 (2019)
Esta Escuela Clásica tiene por postulados:

1. El encontrar sus bases filosóficas en el Derecho Natural.

2. Un respeto absoluto al principio de legalidad.

3. Ver al delito como un ente jurídico y no como un ente filosófico.

4. El libre albedrío

5. La aplicación de las penas a los individuos moralmente responsables.

6. Los que carezcan de libre albedrío como por ejemplo los locos y los niños quedan excluidos del Derecho.

7. La pena es la retribución que se hace al criminal por el mal que hizo en la sociedad.

8. La retribución debe ser exacta.

9. «Las penas son sanciones aflictivas determinadas, ciertas, ejemplares, proporcionales, deben reunir los requisitos de publicidad, certeza, prontitud, fraccionabilidad y reparabilidad, y en su ejecución deben ser correctivas, inmutable e improrrogables».

10. La finalidad de la pena es restablecer el orden social externo que ha sido roto por el delincuente.

11. El Derecho de castigar pertenece al Estado título de tutela jurídica.

12. El Derecho Penal es garantía de libertad, ya que asegura la 
seguridad jurídica ante la autoridad.

13. Se considera que el método debe ser lógico-abstracto, silogístico y deductivo.

\subsection{ESCUELA CLASICA:}

Los positivistas del siglo XIX, bautizaron con el nombre de Escuela Clásica, a todo lo anterior a ellos: a las doctrinas que no se adaptaban a las nuevas ideas, a los recientes sistemas. Bajo la etiqueta de clásicos se suele agrupar a autores y tendencias divergentes en muchos puntos de vista, en algunos casos, inclusive, contradictorias, pero que presentan una serie de concepciones unitarias acerca de postulados fundamentales, que fue lo que permitió a los positivistas reunirlas con propósitos didácticos. El mundo clásico partió de una imagen excelsa, ideal, del ser humano como centro del universo, como dueño y señor absoluto de sí mismo, de sus actos. El dogma de la libertad que hace iguales a todos los hombres (sin diferencias entre el hombre delincuente y no delincuente) y fundamenta la responsabilidad: el absurdo comportamiento delictivo solo puede comprenderse como consecuencia del mal uso de la libertad en una situación concreta, no a pulsiones internas ni a influencias externas. Para los clásicos, el delincuente es una suerte de pecador que optó por el mal, pudiendo y debiendo haber respetado la ley.
Se reconocen como representantes destacados de la escuela clásica del derecho penal, además de Cesare Beccaria, entre otros a Giovanni Carmignani, Pellegrino Rossi y Francisco Carrara. (Quisbert, 2008)

\section{APORTES:}

CESARE BECCARIA (1738 - 1774). Para algunos autores, la criminología clásica, fundada por Cesare Beccaria (1738-1774), trata de encontrar la igualdad de todos los ciudadanos ante la ley y evitar una interpretación desviada por conceptos morales de los juristas o los jueces y, por último, el de limitar el ámbito de las leyes penales al mínimo necesario para disminuir el delito. Se ha afirmado que gracias a la Escuela Clásica se pudo terminar con la barbarie y la injusticia que el derecho penal representaba, procuró la humanización por medio del respeto a la ley, del reconocimiento a las garantías individuales y de la limitación al poder absoluto del Estado. (Alvarez, 2012)

\section{PELLEGRINO ROSSI (1787 - 1848),}

Rossi consideraba que existía un orden moral que todos los seres, libres e inteligentes, deberían de seguir, pensando que aquella tendría que aplicarse en la sociedad, puesto que todos los individuos están hechos para vivir en sociedad: surge un orden obligatorio para toda una sociedad y del cual se derivarían todos los derechos y

Revista Derecho - 5 (2019) 135 
obligaciones. Él piensa que la capacidad de juzgar le pertenece al «superior», pero no niega que todo hombre al ser tal, es inteligente y puede juzgar por igual: para Rosini el fundamento del derecho de castigar, es el eterno principio de la justicia. (Alvarez, 2012)

\section{GIOVANNI CARMIGNANI (1748 -}

1847): Trata de explicar que el castigo que se le impone a un criminal por un delito que cometió, no se hace con el ánimo de tomar una venganza, sino de prevenir que en un futuro no realice otros delitos semejantes. (Alvarez, 2012)

\section{FRANCISCO CARRARA (1805 -} 1888), es considerado por algunos como el padre de la escuela clásica del derecho penal. Este autor sostiene que el derecho es connatural al hombre. La ciencia del derecho criminal es un orden de razones emanadas de la ley moral, preexistente a las leyes humanas. Según Carrara delito es: «La infracción de la ley del estado, promulgada para proteger la seguridad de los ciudadanos, resultante de un acto externo del hombre, positivo o negativo, moralmente imputable o políticamente engañoso». Los postulados de esta escuela son: (Alvarez, 2012)

- $\quad$ La Escuela Positiva se caracteriza por su método científico.

- El delito es un hecho de la naturaleza y debe estudiarse como un ente real, actual y existente.
Su determinismo, es totalmente determinista esta escuela.

Sustituye la responsabilidad moral por la responsabilidad social, puesto que el hombre vive en sociedad y será responsablemente social mientras viva en sociedad. El hecho de que si no hay responsabilidad moral, no quiere decir que se pueden quedar excluido del derecho.

El concepto de Pena se sustituye por el de sanción.

La sanción va de acuerdo a la peligrosidad del criminal.

Estas deben durar mientras dure la peligrosidad del delincuente, y por eso son de duración indeterminada.

La ley penal no restablece el orden jurídico, sino que tiene por misión la de combatir la criminalidad considerada como fenómeno social.

El derecho a imponer sanciones pertenece al Estado a título de defensa social.

Más importante que las penas son los substitutivos penales.

Se acepta «tipos» criminales.

La legislación penal debe estar basada en los estudios antropológicos y sociológicos.

El método es inductivo experimental.

136 Revista Derecho - 5 (2019) 


\subsection{ESCUELAS ECLECTICAS:}

Las críticas contra las escuelas clásica y positivista han dado nacimiento a escuelas eclécticas que mantienen principios de la clásica y toman otros de la positivista, situándose en un término medio. La pena debe ser afianzada con medidas de seguridad. Y respecto a la responsabilidad se conserva el viejo concepto de libre arbitrio, de responsabilidad moral, pero admitiéndose la peligrosidad, temibilidad o estado dañoso para algunos delincuentes.

Esta tendencia dualista de los penalistas críticos o neoclásicos ha influido en la confección de todos los códigos penales promulgados en lo que va transcurriendo del siglo XX. La integración entre derecho penal y criminología requiere entre todo una gran madurez en ambas disciplinas, se recalca sobre la madurez de estas ciencias, porque el derecho penal en aquella época estaba falto de madurez y la criminología empezaba sus primeros pasos.

\section{INCUMPLIMIENTO DE LA} OBLIGACION ALIMENTARIA:

\subsection{MARCO LEGAL}

Artículo $149^{\circ}$ del Código Penal sobre el Incumplimiento de obligación alimentaria: «El que omite cumplir su obligación de prestar los alimentos que establece una resolución judicial será reprimido».

\subsubsection{Definición del delito de Omisión a la Asistencia Familiar:}

«El abandono de familia representa una consecuencia del concepto civil de asistencia familiar, originado por la necesidad de la intervención estatal de garantizar el efectivo cumplimiento de los deberes familiares». (Peña Cabrera, 2008)

El art. $149^{\circ}$ del Código Procesal Penal exige como requisito de procedibilidad para el ejercicio de la acción penal que la obligación alimentaria haya sido establecida mediante resolución judicial firme. Asimismo, la jurisprudencia expresamente viene exigiendo que antes de proceder a la denuncia penal se acredite la notificación al procesado con el apercibimiento expreso de acudir a la vía penal en caso de incumplimiento, de modo tal que si el obligado alimentario no cumple con ejecutar su obligación en el plazo de tres días de notificado el apercibimiento, procederá la denuncia penal correspondiente».

\subsubsection{Definición del Incumplimiento de Obligación Alimentaria:}

Revista Derecho - 5 (2019) 137 
La Constitución Política del Perú, señala «Que no hay prisión por deudas», lo que significaría, según el doctor Bramont Arias y otros, que supondría que el artículo $149^{\circ}$ del Código penal, resultaría inconstitucional, pero este planteamiento es desbaratado por Bernel del Castillo Jesús en su obra «El Delito de Pago de Pensiones», al sustentar que la criminalización de la Omisión a la Asistencia Familiar se da a partir de la presencia de un bien jurídico de gran relevancia, como es la familia, que debe ser protegido por el orden público, porque su asistencia familiar depende del pago alimentario por conceptos de alimentos, vestido, vivienda, salud, educación, capacitación para el trabajo, recreación, es decir, está relacionada con los elementos básicos de supervivencia y siendo la familia el elemento más trascendente del Estado, entonces existen fundamentos sólidos para desbaratar cualquier duda que exista sobre la intervención punitiva en la represión de tal conducta, más aún si el mismo artículo sexto del Texto Constitucional establece que es deber y derecho de los padres alimentar, educar y dar seguridad a sus hijos. El delito de Omisión a la Asistencia Familiar tiene su idea fundamental en la noción de seguridad de los integrantes de la familia, de ahí que el delito que se comete, supone la infracción a los deberes de orden asistencial.

138 Revista Derecho - 5 (2019)

\subsection{CARACTERISTICAS:}

2.2.1. Delito permanente y de peligro: En la Doctrina, el delito en cuestión es una infracción permanente y de peligro, ya que el sujeto pasivo o víctima no tiene la necesidad de probar que con la conducta omisiva del hechor que le haya causado algún perjuicio, pues con la sola puesta en peligro del bien jurídico protegido se perfecciona el ilícito. (Camapana Valderrama, 2013, pág. 74)

2.2.2. E1 tipo omisivo doloso: En nuestra legislación represora se presentan los hechos punibles, como conductas activas, pero estos también pueden ser producidos por el actor sin que solo un solo musculo de su cuerpo se contraiga, por medio de la omisión o de una abstención. (Camapana Valderrama, 2013, pág. 78)

2.2.3. Delito de omisión propia: La doctrina sostiene que es un delito de omisión propia, porque el núcleo del tipo reside en el mero incumplimiento de ciertos deberes, aquellos de asistencia inherente a la patria potestad, la tutela o el matrimonio. Esta caracterización genérica no se destruye porque a tal incumplimiento acompaña o pueda llegar el sujeto activo mediante un hacer positivo, habida cuenta que, por sí mismo, no basta para que pueda considerarse realizada la conducta típica. (Camapana Valderrama, 2013) 
2.2.4. Delito de omisión impropia: La estructura del tipo de estos delitos, también coincide con la omisión propia; se tendrá este tipo de omisión impropia dolosa y omisión impropia culposa. En el primer caso la situación típica será la producción de una lesión o de la puesta en peligro de un bien jurídico, que en este caso son las relaciones de tipo asistencial, el delito se comete cuando se omite prestar los deberes de asistencia a los que le sujeto activo está obligado; en el segundo caso la omisión impropia culposa, del delito sub estudio no cabe considerar culpa cuando el agente de la conducta omisiva no reconoce su posición de garante del bien jurídico protegido, no pudiendo entonces consumarse el delito por este tipo. (Camapana Valderrama, 2013)

EL CÓDIGO PENAL DE 1991. EL DELITO DE OMISIÓN DE ASISTENCIA FAMILIAR. Este delito se encuentra tipificado en el artículo ciento cuarenta y nueve del Código Penal vigente y está estructurado en tres párrafos, que se expone a continuación: «El que omite cumplir su obligación de prestar los alimentos que establece una resolución judicial será reprimido con pena privativa de libertad no mayor de tres años, o con prestación de servicio comunitario de veinte a cincuenta y dos jornadas, sin perjuicio de cumplir el mandato judicial. Si el agente ha simulado otra obligación de alimentos en connivencia con otra persona o renuncia o abandona maliciosamente su trabajo la pena será no menor de uno ni mayor de cuatro años. Si resulta lesión grave o muerte y éstas pudieron ser previstas, la pena será no menor de dos ni mayor de cuatro años en caso de lesión grave, y no menor de tres ni mayor de seis años en caso de muerte.»

La actual legislación penal es de un marcado carácter positivista, sólo persigue la conducta que se halle en desobediencia a la orden judicial de asistencia familiar, ya no contempla la obligación en su estado puro y prístino, ni siquiera como una excepción. Es tal como lo explica Salinas en su obra sobre el Derecho Penal: «...para la configuración del delito en hermenéutica resulta indispensable la pre-existencia de un proceso civil sobre alimentos, en el cual un juez natural ha precisado el deber de asistencia inherente a la institución familiar; de ese modo la obligación de asistencia tiene que ser precisada mediante resolución judicial consentida. Sin previo proceso sobre alimentos es imposible la comisión del ilícito penal de omisión de asistencia familiar.» (Berenguer, 1992)

\section{LA LEY NÚMERO 13906. LEY PUNITIVA DEL ABANDONO}

FAMILIAR: En el Perú, el delito de Omisión a la Asistencia Familiar, se regula con la Ley No. 13906 del 24 de marzo de 1962 bajo el título de Ley de

Revista Derecho - 5 (2019) 139 
Abandono de Familia, actualmente derogada.

Esta ley establecía en su primer artículo el tipo penal consistente en aquel que teniendo la obligación de prestar alimentos a un menor de menos de 18 años de edad, o al mayor incapaz, que está bajo su patria potestad, tutela u otra forma de dependencia, se sustrajera intencionalmente de su cumplimiento. (Berenguer, 1992)

A simple vista pareciera que este tipo fue elaborado bajo una idea iusnaturalista de obligación natural o moral prístina, sin embargo, su artículo $5^{\circ}$ estipula los requisitos de procedencia: a) resolución que señale asignación provisional de alimentos, o sentencia en el juicio correspondiente, y b) que el obligado no haya cumplido su obligación después de haber sido requerido bajo apercibimiento. Es decir está redactado siguiendo los principios de un positivismo estatista por el cual sólo aquello puesto en reconocimiento por el poder público estatal puede ser tutelado penalmente por él. Sin embargo aún no se pude hablar de purismo, el segundo párrafo es prueba de la convivencia yuxtapuesta de estas filosofías pues prescribe la inexigibilidad de estos requisitos si el demandado ha simulado otro proceso de alimentos en connivencia con tercera persona o renuncia o abandona maliciosamente su trabajo, es decir sólo en este caso especial se podía tutelar la obligación natural en su forma prístina sin necesidad de la previa calificación como tal por el Estado. (Berenguer, 1992)

\subsection{RASTREO GENEALOGICO}

\section{EL CASO EN CONCRETO} RESUMEN O NARRACION:

EXPEDIENTE ADM: 018-2016INPE/24-803-SCTP

EXPEDIENTE JUDIC: $\mathrm{N}^{\circ} 0650-2014-$ 75-2101-JR-PE-01.

TIPO DE BENEFICIO: SEMI

\section{LIBERTAD}

INTERNO: Q. A. W. A.

DELITO: INCUMPLIMIENTO DE LA OBLIGACION ALIMENTARIA

En el informe psicológico se detalla el comportamiento durante la permanencia en EP, donde se menciona que el interno se caracteriza por no tener sanciones disciplinarias debido al tiempo de reclusión que era el de apenas 03 meses; durante su vida punitiva se encuentra inscrito en el área de tejido a máquina; la técnica e instrumentos aplicados fueron la entrevista, observación de conducta y un test psicométrico, consta de un examen mental, un inventario de personalidad de Eysenck y el dibujo de una figura humana. El interno luego de ser evaluado recibe orientación y consejería psicológica: no recibe aun psicoterapia cognitiva conductual porque se requiere

140 Revista Derecho - 5 (2019) 
de tiempo para que el interno reconozca comportamientos que lo llevaron a ser recluido en un penal.

En el informe social se llega a la conclusión de que una vez realizada la visita domiciliaria se corrobora el lugar donde domicilia y domiciliará, así como su padre y pareja actual se comprometieron en ayudar para su reinserción a la sociedad. Por otro lado, desarrollará sus actividades laborales como conductor en la Empresa de Transportes San Andrés Apóstol S.A.C., en la entrevista realizada el interno mostró respeto a la autoridad y sus compañeros refieren mantener una convivencia pacífica, por lo que se emite una opinión favorable, siempre y cuando cumpla con las exigencias legales.

En el informe jurídico se logra apreciar que el interno cumple con requisitos establecidos por Ley y no tiene restricciones legales para el beneficio penitenciario. Por lo que el interno cumple el tiempo exigido por Ley para acogerse a tal beneficio de Semi Libertad al haber acumulado un tercio de la pena impuesta con carcelería efectiva.

\subsection{TABLA DE LAS ESCUELAS JURIDICO PENALES}

TABLA $N^{\circ}$ 1: Escuelas jurídico penales y características.

\begin{tabular}{|c|c|}
\hline $\begin{array}{c}\text { ESCUELA } \\
\text { CLASICA }\end{array}$ & $\begin{array}{l}\text { - Libre albedrío } \\
\text { - Igualdad de derechos } \\
\text { - Responsabilidad moral } \\
\text { - Objeto: el delito (jurídico) } \\
\text { - Método: deductivo (especulativo) } \\
\text { - Pena proporcional al delito } \\
\text { - Clasificación de delincuentes }\end{array}$ \\
\hline $\begin{array}{l}\text { ESCUELA } \\
\text { POSITIVA }\end{array}$ & $\begin{array}{l}\text { - Negación del libre albedrio } \\
\text { - Responsabilidad social } \\
\text { - Objeto: el delincuente } \\
\text { - Método: inductivo (experimental) } \\
\text { - Pena: proporcional a la peligrosidad } \\
\text { - Prevención más que represión } \\
\text { - Medidas de seguridad } \\
\text { - Clasificación de delincuentes }\end{array}$ \\
\hline $\begin{array}{c}\text { ESCUELA } \\
\text { ECLECTICA }\end{array}$ & $\begin{array}{l}\text { TS - No opta por el determinismo } \\
\text { radical del positivismo ni por el } \\
\text { libre albedrí absoluto del } \\
\text { clasicismo. Conserva la idea de la } \\
\text { "RESPONSABILIDAD MORAL" } \\
\text { como fundamento de la pena, y la } \\
\text { "temibilidad" o "peligrosidad". } \\
\text { TS - Pena y medidas de seguridad } \\
\text { JE - Pragmatismo } \\
\text { JE - Estado de peligro } \\
\text { JE - Delito como fenómeno natural y } \\
\text { jurídico. } \\
\text { JE - Libre albedrio, posición } \\
\text { intermedia } \\
\text { JE - La pena es la defensa social } \\
\text { JE - Penas y medidas de seguridad } \\
\text { JE - Delincuentes (normales y } \\
\text { anormales) }\end{array}$ \\
\hline
\end{tabular}

FUENTE: Elaboración grupal. 
De acuerdo a la tabla $\mathrm{N}^{\circ} 1$ podemos observar que la escuela clásica muestra diferentes características, tales como el libre albedrío, igualdad de derechos, responsabilidad moral. El objeto para ésta escuela jurídico penal es el delito y su método es inductivo, la pena es proporcional al delito y se hace una clasificación de delincuentes, como se pudo analizar en el expediente del interno y en cuanto a la entrevista realizada al Dr. José Luis Ticona Yanqui, podemos acotar de que ésta escuela concuerda con muchas de las características ya mencionadas, ya que la persona al actuar con el libre albedrio podría obtener consecuencias por tal conducta, es tal el caso del interno de iniciales Q.A.W.A que al no cumplir con la obligación de pasar alimentos a su menor hijo, éste fue demandado y recluido en el centro penitenciario, el Dr. Ticona nos indica en la entrevista de que nadie puede privársele de su derecho de libertad, por el hecho de deudas, sin embargo cree que acertadamente la constitución ha previsto que en el caso de deudas por pensiones de alimentos, las personas que se encuentran dentro de ese supuesto puedan ser privadas de su libertad. Personalmente hace mención, de que le parece pertinente por cuanto se ha producido un hecho de connotación social exagerada. En algunos casos de que muchos padres de familia generalmente varones, no cumplen con sus menores hijos en el tema de su alimentación de su vestido y educación, y una forma de coaccionarlos al cumplimiento del mismo, más allá del cumplimiento de cumplir con su deber de padre, cree que ha sido el Estado utilice este medio de coerción social, por lo menos, a él le parece pertinente.

\section{CONCLUSIONES:}

El delito de incumplimiento de la obligación alimentaria es un problema que existe en todos los estatus sociales de nuestra sociedad, pero se da con mayor frecuencia en los estatus socio económico menos favorecidos.

Es muy pertinente y adecuado que las personas sancionadas por el delito de incumplimiento de obligación alimentaria sean penalizadas, por cuanto se produce un hecho de connotación social muy grave; esto en cuanto que muchas familias no cumplen con su deber y obligación para con los integrantes del núcleo familiar en este caso con los menores de edad.

A causa de no conocer las consecuencias que trae consigo el incumplimiento de la obligación alimentaria, es que muchas de las personas tales como el caso de estudio que hemos abarcado se encuentran recluidas privadas de su libertad. Es por esto, que la educación que se recibe desde pequeños es imprescindible dentro de la formación de cada persona. 
Este delito, es entendido como el abandono familiar que representa una consecuencia del concepto civil de asistencia familiar, dejando al margen el concepto de familia que debe ser protegida de una manera rigurosa desde la base del ordenamiento jurídico.

\section{REFERENCIAS:}

Alvarez, G. M. (2012). Apuntes acerca de dos escuelas criminológicas: Clásica y Positivista. México: Facultad de Psicología.

Amucharegui, I. G. (2006). Derecho Penal. Mexico: Editorial Harla. Mexico.

Berenguer, R. (1992). Las corrientes filosóficas en la legislacion peruana sobre el delito de la omisión a la asistencia familiar en la modalidad de incumplimiento de obligacion a prestar los alimentos.

Camapana Valderrama, M. (2013). El Delito de Omisión a la Asistencia Familiar. Lima: Editorial Universidad Inca Garcilaso de la Vega; Lima, Perú.
Elbert, C. A. (2001). Manual básico de criminología. Buenos Aires: Eudeba.

Ferri, E. (1887). Los nuevos horizontes del Derecho y del Procedimiento Penal. Madrid: Centro Editorial de Gongora. España, Madrid.

Ferri, E. (1933). Principio de Derecho Criminal. Madrid: Editorial Reus. Madrid, España.

Jiménez de Asua, L. (1950). Tratado de Derecho Penal, tomo II. Buenos Aires.

Peña Cabrera, R. (2008). Tratado de Derecho Penal, Parte especial. Lima: Ediciones Juridicas. LIma, Peru.

Quisbert, E. (2008). Historia Del Derecho Penal A Través De Las Escuelas. La Paz: Centro de Estudios de Derecho.

Sainz, C. J. (1990). Lecciones de Derecho Penal, Parte General. Barcelona: Bosch. 
144 Revista Derecho - 5 (2019) 